\title{
Effectiveness of transperforation myringoplasty in bilateral chronic otitis media patients undergoing contralateral tympanoplasty
}

\author{
A T IHSAN, K RANJANA \\ Department of Otorhinolaryngology and Head and Neck Surgery, Jubilee Mission Medical College and Research \\ Institute, Thrissur, India
}

\begin{abstract}
Objectives: Bilateral tympanic membrane perforation closure is usually performed by otosurgeons in two sittings. However, in this study, transperforation myringoplasty was performed alongside contralateral tympanoplasty in a single sitting. The effectiveness of transperforation myringoplasty procedure and the benefits of single sitting bilateral surgery were evaluated.

Methods: A prospective study of 50 selected patients with mucosal-type bilateral chronic otitis media was conducted. All patients underwent transperforation myringoplasty on the side that met the inclusion criteria and tympanoplasty on the contralateral side. Graft uptake and hearing improvement were evaluated after 6 months.

Results: At the 6-month follow up, the graft uptake rate was 82 per cent, the hearing gain was $11.5 \mathrm{~dB}$ and the air-bone gap gain was $11.6 \mathrm{~dB}$.

Conclusion: This procedure offers perforation closure in a single sitting to patients with bilateral chronic otitis media who meet the inclusion criteria.
\end{abstract}

Key words: Myringoplasty; Tympanic Membrane Perforation; Tympanoplasty

\section{Introduction}

Patients with bilateral mucosal-type chronic otitis media are commonly seen in general practice, usually presenting with intermittent ear discharge and hearing loss. Such patients usually undergo tympanoplasty in two sittings with a minimum interval of six months. This approach reduces the risks of graft failure and iatrogenic sensorineural hearing loss. There are few reports of single sitting bilateral surgery.

In transperforation myringoplasty, a defect in the tympanic membrane is closed via the perforation using a transcanal approach without tympanomeatal flap elevation. In the present study, selected patients underwent simultaneous bilateral surgery comprising tympanoplasty with cortical mastoidectomy on the side with more severe disease (i.e. the contralateral side) and transperforation myringoplasty on the socalled inactive side (i.e. the study side). All patients were evaluated at the six-month follow up.

\section{Materials and methods}

This prospective study included 50 patients with bilateral mucosal-type chronic otitis media who attended the out-patient department of a tertiary care hospital from December 2012 to November 2014.

Inclusion criteria were: (1) the patient should be willing to undergo concomitant bilateral ear surgery; (2) the tympanic membrane has a small to mediumsized central perforation with no active discharge from the middle ear on one side; (3) pure tone audiometry shows a hearing threshold of $40 \mathrm{~dB}$ or less (to rule out ossicular problems); and (4) there should be no active disease in the nose. Exclusion criteria were: (1) a very narrow external auditory canal, impairing transcanal visualisation of the perforation; (2) the tympanic membrane shows subtotal perforation, a marginal perforation, an attic perforation and atticoantral disease, or active discharge from the middle ear; and (3) a hearing threshold of more than $40 \mathrm{~dB}$.

Written informed consent was obtained from all patients who met the inclusion criteria and were willing to participate in the study. A detailed medical history was taken for each patient, followed by a thorough microscopic examination of the ears. All findings were recorded systematically. Symptoms on the study side were ear discharge, either alone ( 24 per cent) or combined with hearing loss ( 76 per cent). 
Patients with a history of ear discharge could have had either a wet or dry ear on the study side at presentation. For patients with dry ears, surgery was scheduled within 1-2 months; in contrast, patients with wet ears were given a course of antibiotics (7-14 days) and kept under observation for 2-3 months. If the ear on the study side was then found to be dry, bilateral surgery was scheduled for these patients.

All surgery was performed under general anaesthesia by the same otosurgeon (the senior author). A post-auricular incision was first made in the contralateral ear and a graft large enough for bilateral grafting was harvested and prepared. After completing standard tympanoplasty on the contralateral side, the study side was prepared for transperforation myringoplasty. The microscope was adjusted to focus on the perforation and visibility of the entire perforation margin was confirmed (Figure 1). The margin was trimmed and freshened using a curved needle and cup forceps (Figure 2). The undersurface of the drum remnant was abraded with a round knife. The entire middle ear was then filled with Gelfoam ${ }^{\circledR}$ (impregnated with antibiotic solution) and a graft bed was created (Figure 3). The fascia graft was cut to the correct size and shape, and then carefully placed medial to the drum remnant to completely cover the perforation. By gentle manipulation with an elevator, the graft was tucked under the drum remnant, which was then evened out with a gimmick (annulus elevator; Figures 4 and 5). The Gelfoam ${ }^{\circledR}$ was kept over the graft and covered with a light antibiotic ointment pack for support. All patients were given systemic antibiotics peri-operatively. The antibiotic pack was removed after a week.

Patients were followed up after one week, one month, two months and six months. Anatomical and functional

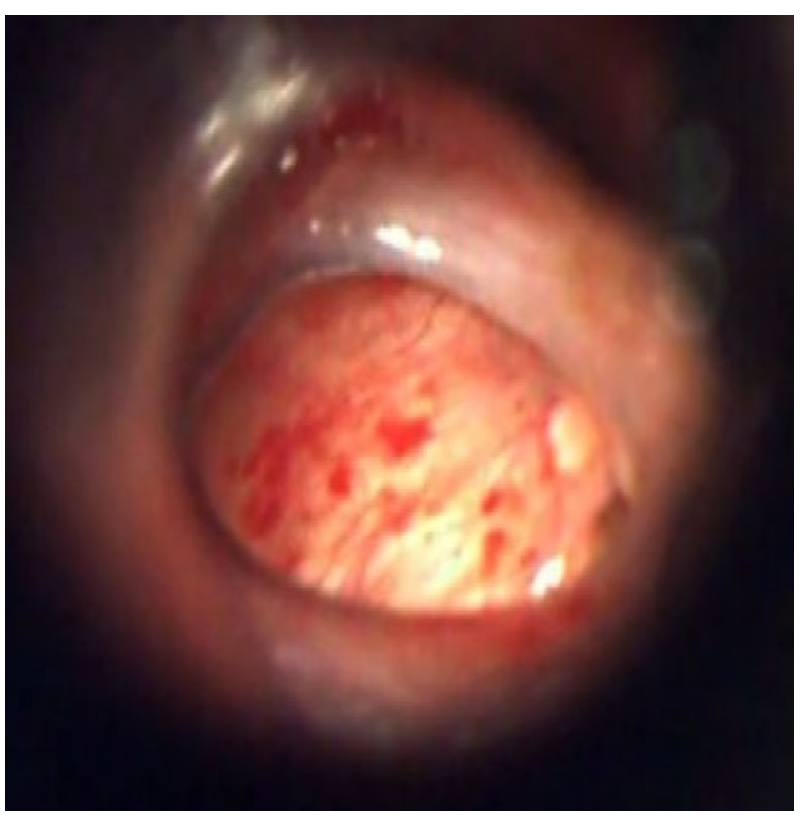

FIG. 1

Photograph showing a medium-sized perforation with visible margins.

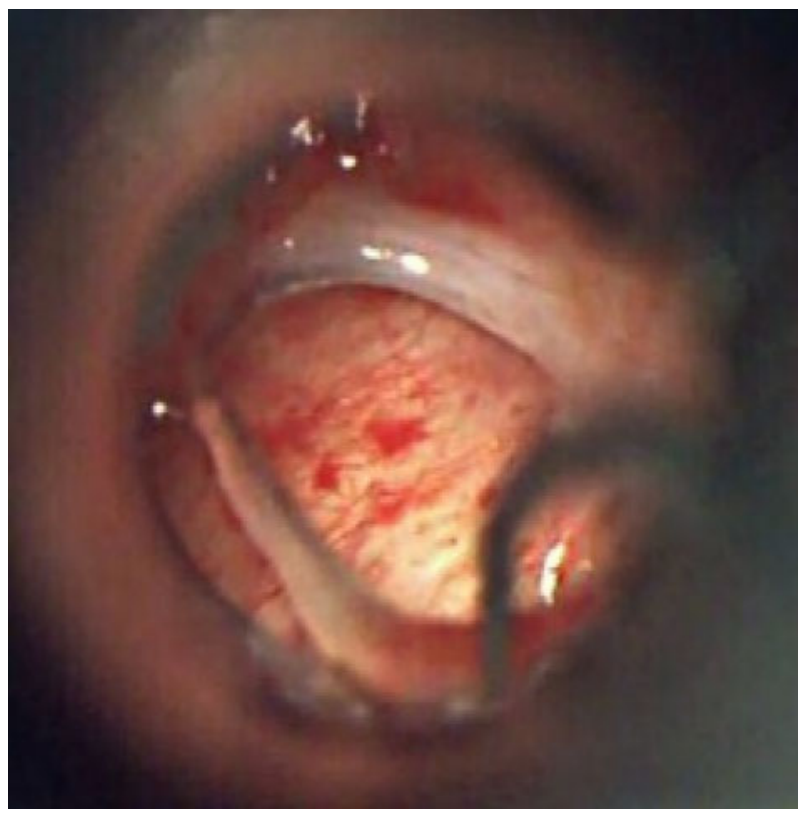

FIG. 2

Photograph showing freshening of perforation margins.

improvements after 6 months were analysed using IBM SPSS Statistics software version 22.0 (Armonk, New York, USA).

\section{Results}

Graft uptake was successful in 86 per cent of patients (43 out of 50) after 3 months and in 82 per cent (41 out of 50) after 6 months. Both of the patients who lost the graft after three months had developed an infection and tympanic membrane perforation.

On the study side, the mean pre-operative pure tone average (PTA) was $28.6 \mathrm{~dB}$ (range 20.0-40.0 dB) and

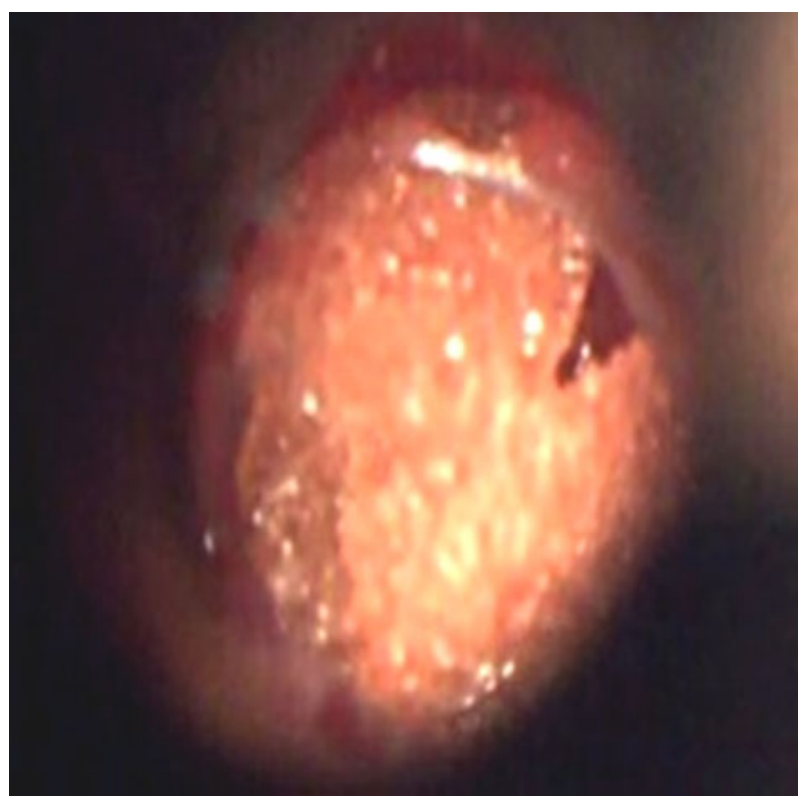

FIG. 3

Photograph showing the prepared graft bed. 


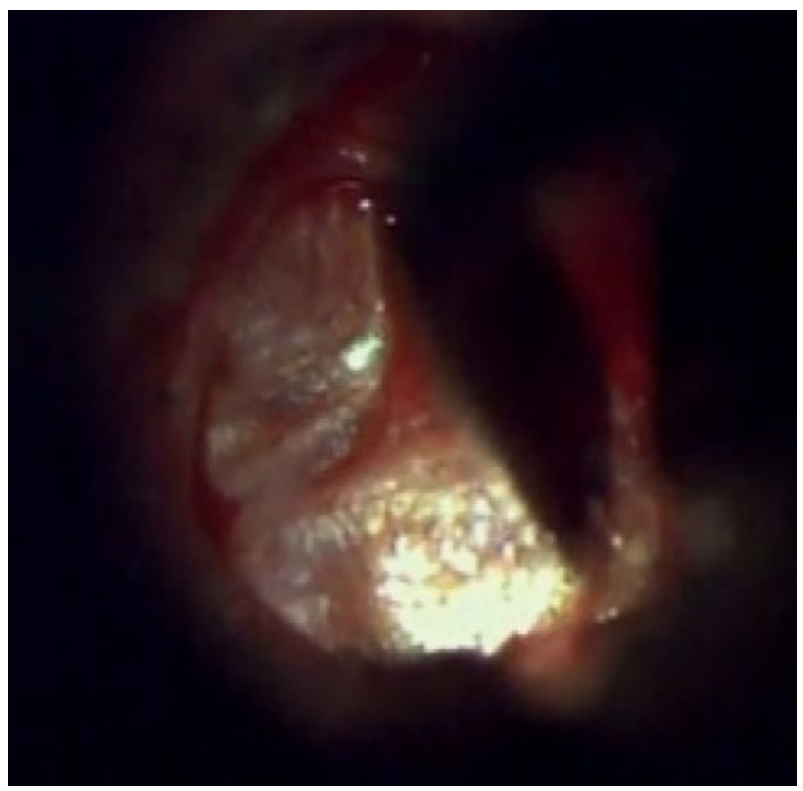

FIG. 4

Photograph showing graft tucking with a annulus elevator.

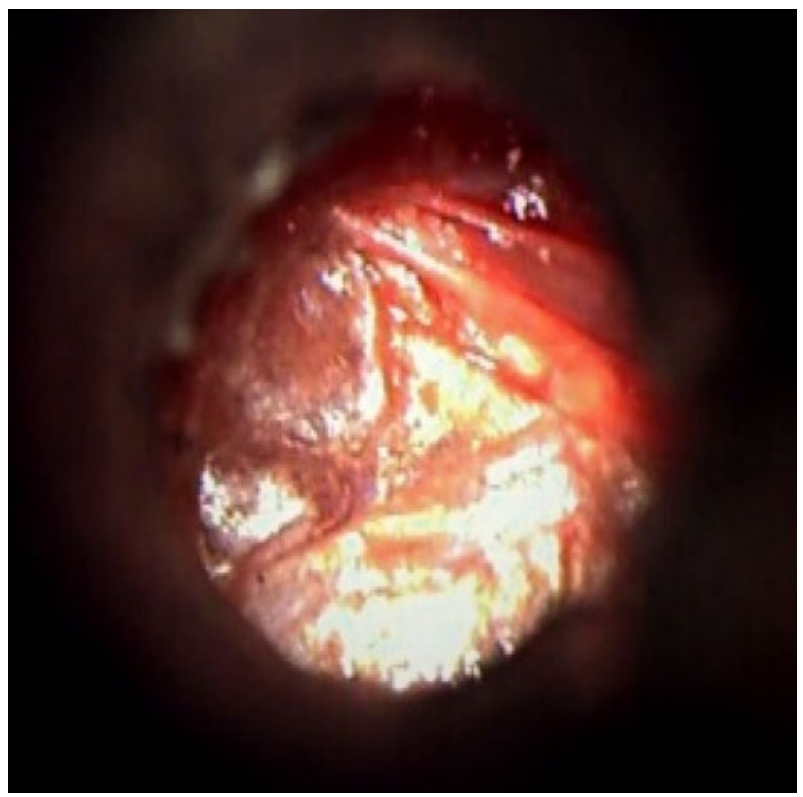

FIG. 5

Photograph showing the underlay graft in position.

the mean post-operative PTA at 6 months was $17.1 \mathrm{~dB}$ (range 11.5-40.0 dB; Table I). The post-operative improvement in hearing thresholds was significant $(p=0.004)$. The mean hearing gain, calculated as the difference between the pre- and post-operative mean PTA values (28.6 dB and $17.1 \mathrm{~dB}$, respectively) was $11.5 \mathrm{~dB}$

The mean pre-operative air-bone gap (ABG) was $14.0 \mathrm{~dB}$ (range 5.0-30.0 dB; Table II). Post-operatively, the ABG was closed in 82 per cent of patients and within $10 \mathrm{~dB}$ in 92 per cent. The ABG gain, calculated as the difference between the mean pre- and
TABLE I

PRE- AND POST-OPERATIVE PURE TONE AVERAGES

\begin{tabular}{lcc}
\hline PTA range $(\mathrm{dB})$ & \multicolumn{2}{c}{ Patients $(n)$} \\
\cline { 2 - 3 } & Pre-op & Post-op \\
\hline$\leq 15$ & 0 & 34 \\
$16-19$ & 0 & 4 \\
$20-25$ & 20 & 8 \\
$26-30$ & 14 & 2 \\
$31-35$ & 10 & 1 \\
$36-40$ & 6 & 1 \\
\hline
\end{tabular}

$\mathrm{PTA}=$ pure tone average; Pre-op $=$ pre-operatively; post-op $=$ post-operatively

TABLE II

PRE- AND POST-OPERATIVE AIR-BONE GAPS

\begin{tabular}{lcc}
\hline ABG range $(\mathrm{dB})$ & \multicolumn{2}{c}{ Patients $(n)$} \\
\cline { 2 - 3 } & Pre-op & Post-op \\
\hline 0 & 0 & 41 \\
$5-10$ & 18 & 3 \\
$11-20$ & 25 & 6 \\
$21-30$ & 7 & 0 \\
\hline
\end{tabular}

$\mathrm{ABG}=$ air-bone gap; Pre-op $=$ pre-operatively; post-op $=$ postoperatively

post-operative $\mathrm{ABG}$ values $(14.0 \mathrm{~dB}$ and $2.4 \mathrm{~dB}$, respectively), was $11.6 \mathrm{~dB}$.

At the end of follow up, all patients with pre-operative pure conductive loss and post-operative graft closure $(n=38)$ recovered normal hearing with a PTA value of less than or equal to $15 \mathrm{~dB}$. The other patients with successful graft uptake $(n=4)$ had higher PTA values due to a sensorineural component that was present before surgery. Patients with successful graft uptake (82 per cent) had full ABG closure after six months. The graft failure group comprised four patients with unchanged $\mathrm{ABG}$ and five with $\mathrm{ABG}$ closure of $3-8 \mathrm{~dB}$.

In patients who underwent unilateral tympanoplasty performed by the same surgeon and during the same period, the graft uptake rate was 94-98 per cent. In the present study, the graft uptake rate of 96 per cent on the contralateral side shows that performing surgery on both ears in a single sitting does not reduce the uptake rate for the study side.

In the graft failure group, the defect was present in either the posterosuperior or anterosuperior aspect, possibly due to medialisation of the graft. For most patients, the perforation size was decreased which reduced the amount of middle-ear mucosa exposed to the external environment. Further, hearing was improved and there was a lower incidence of ear discharge postoperatively.

Cost and time savings for patients who underwent single sitting surgery with successful graft uptake were compared with those associated with undergoing tympanoplasty at a later date (i.e. in two sittings). At the 
Jubilee Mission Medical College and Research Institute, the cost of transperforation myringoplasty is around 12 per cent of the cost of tympanoplasty because it is performed as combination surgery using a simpler technique. Therefore, if this treatment is successful, patients save 88 per cent of the cost of second sitting tympanoplasty and avoid the additional costs of medication and a second hospital stay. However, those with graft failure had a financial loss of 12 per cent of the tympanoplasty cost. In this study, none of the patients with graft failure was upset about losing money because they considered this a small price to pay for attempting this surgical approach. Most were satisfied with their post-operative hearing improvement and absence of ear discharge.

In this study, all transperforation myringoplasty procedures were performed by the same surgeon and completed in around 20 minutes. The same surgeon takes around 60 minutes for a tympanoplasty procedure. Thus, single sitting surgery saves the time needed to take a separate graft on the myringoplasty side.

\section{Discussion}

Bilateral chronic otitis media is considered a double burden for patients because they have to undergo two hospital stays and face additional risks to obtain a dry ear and improved hearing. In general, otosurgeons are reluctant to perform bilateral surgery in a single sitting because of the longer surgical duration, difficulty in changing the position of the surgical team (i.e. surgeon and assistants) and equipment, and the reported risk of iatrogenic sensorineural hearing loss. However, on balance, simultaneous transperforation myringoplasty on the side less affected by disease could be effective for selected patients with bilateral chronic otitis media.

A literature review identified several reports of similar transperforation myringoplasty techniques (Table III). All procedures were performed without tympanomeatal flap elevation and via different routes, with different grafts and with slight variations in procedure. All reports that included similar graft placement were for unilateral procedures: most bilateral procedures were type 1 tympanoplasty (Table IV).

Sharma et al. reported outcomes for 25 patients (50 ears) who opted for single sitting bilateral myringoplasty using mini-endaural and permeatal routes with or without a tympanomeatal flap, and another group of 25

\begin{tabular}{|c|c|c|}
\hline SINGLE SITTING B & $\begin{array}{l}\text { TABLE IV } \\
\text { ERAL TYMP } \\
\text { TAKE RATE }\end{array}$ & NOPLASTY: GRAFT \\
\hline Study & Patients $(n)^{*}$ & Graft uptake rate $(\%)$ \\
\hline Sharma et al. ${ }^{6}$ & 25 & 90 \\
\hline Caye-Thomasen et al. & 26 & 94 \\
\hline El-Ahl et al. ${ }^{8}$ & 30 & 93 \\
\hline Mane et al. ${ }^{9}$ & 14 & 96 \\
\hline Ansari et al. ${ }^{10}$ & 30 & 93 \\
\hline Rai et al. ${ }^{11}$ & 30 & 93 \\
\hline Wang et al. ${ }^{12}$ & 22 & 93 \\
\hline
\end{tabular}

*Note that the number of ears is twice the number of patients in all studies.

patients (25 ears) who opted for unilateral myringoplasty by the post-auricular route. ${ }^{6}$ In all patients, temporal fascia was used for grafting by the underlay technique. Perforation closure was successful in 90 per cent of ears after single sitting bilateral myringoplasty and in 88 per cent of ears in the unilateral myringoplasty group. Caye-Thomasen et al. reported outcomes for 26 patients (52 ears) who underwent bilateral myringoplasty and type I tympanoplasty. ${ }^{7}$ Surgery was performed via a transcanal approach in all ears except one, the onlay technique was used most frequently ( 83 per cent), and the graft material was fascia (56 per cent), tragal perichondrium (38 per cent) or cartilage palisades (6 per cent). Perforation closure was achieved in 49 out of 52 ears ( 94 per cent).

El-Ahl et al. operated on 30 patients (60 ears), with successful perforation closure in 56 ears ( 93.3 per cent) and residual perforation in the other 4 ears. $^{8}$ In all, 26 patients had successful bilateral graft uptake. Mane et al. reported outcomes for 14 patients (28 ears) who underwent bilateral type I tympanoplasty. ${ }^{9}$ A postaural approach was used for 5 ears, and an endaural or endomeatal approach was used for the other 23 . The underlay technique was used for all ears: the graft material was fascia lata for 10 patients and temporalis fascia for 4 . The overall graft uptake rate was 96 per cent. Ansari et al. reported the outcomes for 30 patients who underwent bilateral type I tympanoplasty: successful closure of the tympanic membrane perforation was achieved in 56 out of 60 ears. ${ }^{10}$

Rai et al. assessed the surgical outcomes for 60 patients, of whom 30 had unilateral disease and 30 had bilateral diseaase. ${ }^{11}$ All patients underwent type I tympanoplasty using the post-auricular inlay technique using a temporalis fascia graft. Graft uptake 
rates of 90 per cent and 93 per cent were achieved in the unilateral and bilateral surgery groups, respectively. Wang et al. reported the outcomes of bilateral single sitting myringoplasty in 22 patients, along with 40 monoaural control patients. ${ }^{12}$ The closure rate for tympanic membrane perforations was 93.2 per cent (41 out of 44 ears).

Kim et al. compared three subgroups of patients who underwent bilateral mastoidectomy, unilateral mastoidectomy with contralateral tympanoplasty, and bilateral tympanoplasty. ${ }^{13}$ A total of 85 patients with chronic otitis media or cholesteatoma underwent bilateral single sitting surgery. The results of hearing tests did not differ significantly among the three groups except for hearing gain in the bilateral tympanoplasty group $v s$ the contralateral tympanoplasty group $(p=0.033)$. The graft uptake rate for single sitting bilateral surgery was 90-96 per cent, which is higher than the 82 per cent success rate achieved in the present study. The higher success rate may be due to tympanomeatal flap elevation, which allows a more meticulous procedure. Despite this, the present study reported the second highest number of single sitting bilateral ear surgical procedures so far, next to Kim et al. ${ }^{13}$

- This prospective study included 50 bilateral chronic otitis media patients

- Bilateral tympanic membrane perforation closure was performed in a single sitting via simultaneous transperforation myringoplasty and contralateral tympanoplasty

- The graft uptake rate was 82 per cent

- Significant hearing improvement and air-bone gap closure were achieved

- Bilateral perforation closure in a single sitting can reduce costs, time taken off work and travelling time for suitable patients

The present study had the following notable findings. Firstly, transperforation myringoplasty involves minimal manipulation of the middle ear, which minimises the peri-operative injury risk. Hence, even if there is an element of sensorineural hearing loss (as reported for bilateral cases), the risk is definitely reduced. Secondly, even if the graft fails, there is still a chance for tympanoplasty at a second sitting. In the present study, the two patients with graft failure opted for a second surgical procedure during the study period. Since tympanomeatal flap elevation had not been performed in these patients, there was no tissue fibrosis and revision surgery was therefore as straightforward as in a fresh case. More importantly, no waiting period was needed before performing tympanoplasty on the same side. Thirdly, the procedure is relatively simple and does not require a high level of surgical skill. It can be completed within 20 minutes using graft material harvested and kept for tympanoplasty on the other side. Fourthly, operative and post-operative morbidity is reduced. A post-aural incision for graft harvesting is made on only one side; hence, no incision or heavy dressing is needed on the transperforation side. Further, only a small external auditory canal pack is necessary, and this can be removed after a week. All patients in this study found that hearing impairment due to the bilateral ear pack was modest and acceptable. Finally, bilateral procedures can be performed in a single sitting, which avoids a second exposure to anaesthesia and a hospital stay, reduces the length of absence from work, and reduces the burden on the healthcare system.

With the growing use of endoscopic ear surgery, simultaneous bilateral procedures may become more popular among both surgeons and patients.

References

1 Yuasa Y, Yuasa R. Postoperative results of simple underlay myringoplasty in better hearing ears. Acta Otolaryngol 2008;128: 139-43

2 Chen C, Chiu W, Yu J, Lee Y, Chang K. Transcanal myringoplasty with perichondrium. Otolaryngol Head Neck Surg 2011;141:199

3 Singh GB, Sharma A, Singh N. Role of transtympanic myringoplasty in modern otology. J Otolaryngol 2006;35:408-12

4 Albera R, Riontino E, Milan F, Lacilla M, Gervasio CF, Cavalot AL et al. Transmeatal underlay myringoplasty. Indications and results. Otorinolaringologia 1999;49:11-15

5 Calderón RM, Meléndez PC, Morales SC, Torres MD. Experience in transcanal myringoplasty with inlay tragal perichondrium. Rev Otorrinolaringol Cir Cabeza Cuello 2012;72:151-6

6 Sharma RC, Saroch M. Our experience with single sitting bilateral myringoplasty. Indian J Otol 2013;19:59-61

7 Caye-Thomasen P, Nielsen TR, Tos M. Bilateral myringoplasty in chronic otitis media. Laryngoscope 2007;117:903-6

8 El-Ahl MAS, Amer HS, El-Anwar MW. Simultaneous bilateral myringoplasty as a single-stage operation. Egypt J Otolaryngol 2013;29:16-19

9 Mane R, Patil B, Mohite A, Varute VV. Bilateral type 1 tympanoplasty in chronic otitis media. Indian $J$ Otolaryngol Head Neck Surg 2013;65:293-7

10 Ansari MA, Khayani IA, Farrukh MS, Kashmiri ZA, Farooq MU. Outcome of bilateral myringoplasty in dry central perforation: an appraisal. J Dow Univ Health Sci 2014;8:16-20

11 Rai AK, Singh GB, Sahu R, Singh S, Arora R. Evaluation of simultaneous bilateral same day tympanoplasty type I in chronic suppurative otitis media. Auris Nasus Larynx 2014;41: 148-52

12 Wang HB, Yu F, Shan XZ, Zhang F, Zhao LZ, Long SB et al. Clinical analysis of bilateral same-day myringoplasty for 22 cases with bilateral tympanic membrane perforation. Zhonghua Er Bi Yan Hou Tou Jing Wai Ke Za Zhi 2013;48:329-31

13 Kim CW, Lee JS, Park CH, Kwon SY, Kim DK, Lee JH. Comparison of sequential same-day middle ear surgeries: bilateral mastoidectomy, unilateral mastoidectomy with contra lateral tympanoplasty, and bilateral tympanoplasty. Eur Arch Otorhinolaryngol 2015;272:1395-402

Address for correspondence:

Dr A T Ihsan,

Department of Otorhinolaryngology and Head and Neck Surgery, Jubilee Mission Medical College and Research Institute,

Thrissur,

Kerala 680005, India

E-mail: Ihsanent@gmail.com

Dr A T Ihsan takes responsibility for the integrity of the content of the paper

Competing interests: None declared 